\title{
Mesothelioma Driver Genes, Ferroptosis, and Therapy
}

\author{
Emanuela Felley-Bosco ${ }^{1 *}$ and Steven G. Gray ${ }^{2}$ \\ ${ }^{1}$ Laboratory of Molecular Oncology, Department of Thoracic Surgery, University Hospital Zurich, Zurich, Switzerland, \\ ${ }^{2}$ Thoracic Oncology Research Group, Trinity Translational Medical Institute, St. James's Hospital, Dublin, Ireland
}

Keywords: mesothelioma, ferroptosis, regulated cell death, NF2, BAP1

If a given cell has a propensity to die in a certain manner, the logical step for this cell to become a cancer cell is to insure its survival by installing mechanisms circumventing the predestined regulated cell death. A clear example of this occurs in follicular lymphoma where chromosomal re-arrangements result in $\mathrm{Bcl} 2$ overexpression, allowing escape from apoptosis and tolerance to undesired generation of otherwise physiological mutations and double strand breaks necessary to produce the variability necessary for antigen recognition site by immunoglobulin (1).

The predestined regulated cell death mechanism for mesothelial cells is not known, but recent data have linked two frequent drivers of mesothelioma, NF2 and $B A P 1(2,3)$, to ferroptosis $(4,5)$. The latter is a more recently described type of iron-dependent regulated cell death (6).

An additional driver of mesothelioma, which is however less specific to this cancer type, is loss of CDKN2A (7-9). One of the products encoded by CDKN2A gene is p16, which is one of the effectors of senescence (10). The latter is a state of stable cell cycle arrest with active metabolism where resistance to ferroptosis induction has been observed due to decreased iron bioavailability, linked to increased ferritin (FTH1) levels, and accompanied by increased levels of iron regulatory protein 2 (IREB2) and decreased levels of iron-cluster assembly enzyme (ISCU) (11).

The aim of this Opinion paper is to complement the editorial by Fennell (12) with some

Glen Reid,

University of Otago, New Zealand

Reviewed by: Yoshitaka Sekido,

Aichi Cancer Center, Japan

Thomas John,

Austin Health, Australia

${ }^{*}$ Correspondence:

Emanuela Felley-Bosco emanuela.felley-bosco@usz.ch

Specialty section:

This article was submitted to

Thoracic Oncology,

a section of the journal

Frontiers in Oncology

Received: 13 September 2019 Accepted: 12 November 2019 Published: 27 November 2019 additional considerations, which include potential ideas regarding treatment, based on data from our own model of mesothelioma development (13) and the mesothelioma TCGA database (3).

In ferroptosis (Figure 1A), cell death is executed by reactive oxygen species (ROS)-mediated peroxidation of polyunsaturated fatty acids (PUFAs). The origin of ROS includes incomplete reduction of oxygen during electron transport to form superoxide, and a direct generation of superoxide by the membrane bound NADPH oxidases (NOX) (14). Lipid peroxidation is prevented by glutathione peroxidase 4 (GPX4), which uses glutathione (GSH) as reducing agent [reviewed in (15)]. GSH is synthesized from cysteine, which is either derived from methionine through methionine-R-sulfide reductase B2 (MSRB2), or it is imported. Interestingly, MRSB2 expression is significantly higher in epithelioid compared to tissues with a sarcomatoid molecular profile (2). Import of cysteine is mediated by SLC7A10 transporter, but cysteine can also be derived from the reduction of cystine (product of the oxidation of two cysteine molecules, which are then linked via a disulfide bond). Cystine is transported into the cell through the system $\mathrm{Xc}^{-}$transporter, which includes SLC7A11 subunit. It is worth noting that only cystine is present in cell culture medium, and, as for cells like lymphocytes [reviewed in (16)], mesothelial and mesothelioma primary cells grow better in the presence of beta-mercaptoethanol $(17,18)$. This effect is likely due to formation of beta-mercaptoethanol dimers with cystine facilitating its uptake by other transporters (19).

BAP1 decreases the expression of SLC7A11 (5), leading to increased sensitivity to ROS and erastin in mesothelioma cells.

PUFA abundance, and hence predisposition to ferroptosis, is dependent on the expression of acyl-CoA synthetase long-chain family member 4 (ACSL4). In the absence of a negative control downstream NF2/Hippo pathway, the transcriptional co-activator YAP increases ACSL4 expression (4). Resistance to ferroptosis is associated with high expression levels of aldo-keto reductase 
A

B

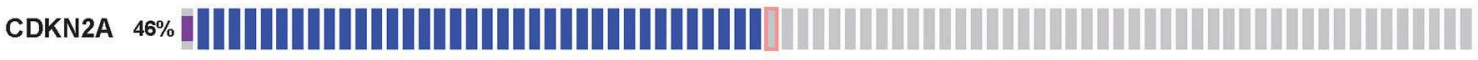
BAP1 60\%

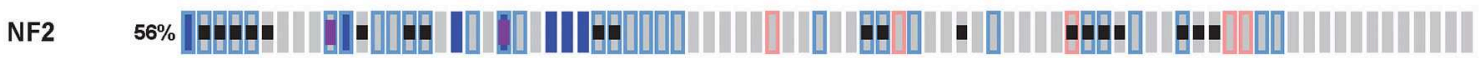

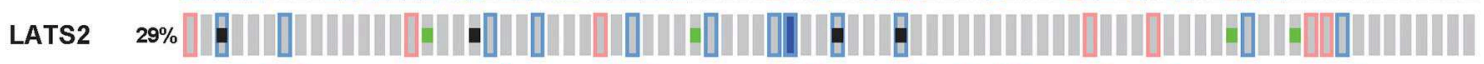
GPX4 23\% SLC7A11 4\% SLC7A10 9\%

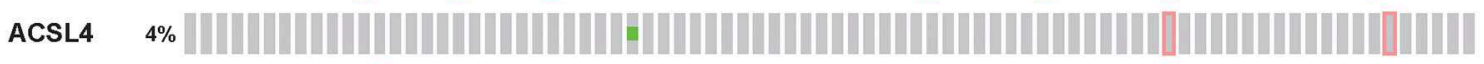

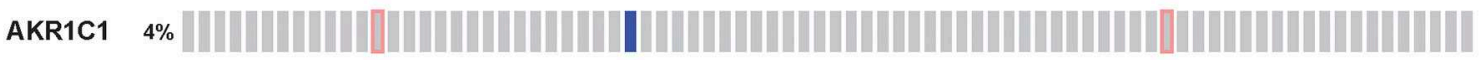

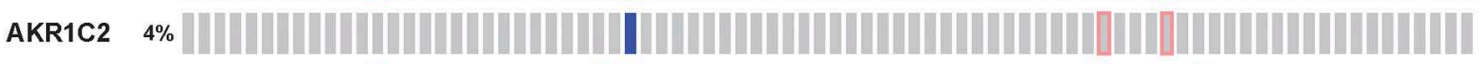

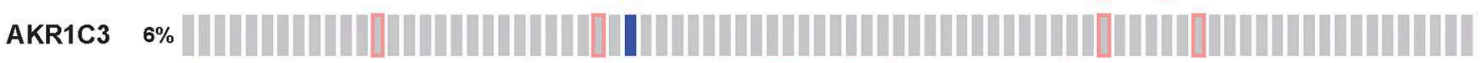

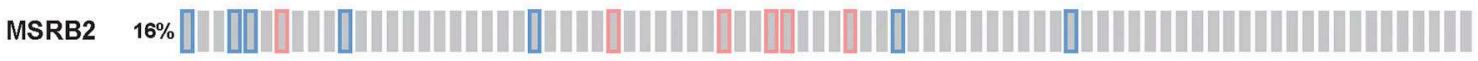
Nox1 11\%

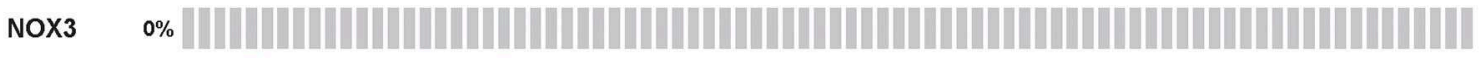

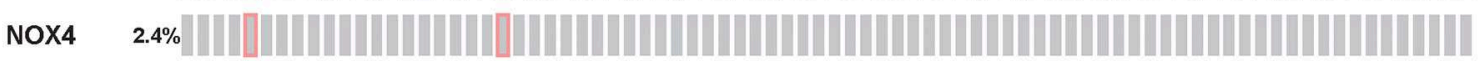
Nox5 $\quad$ 10\%

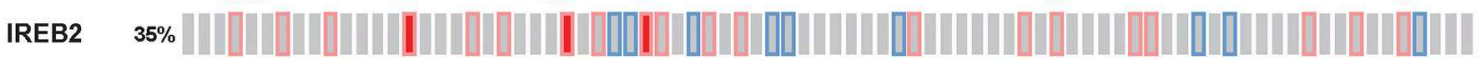

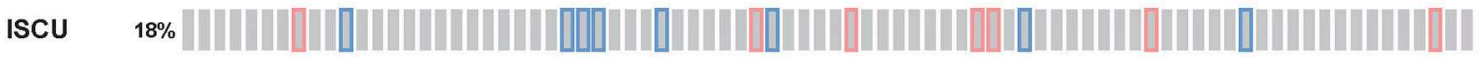
Fтн1 20\%

FIGURE 1 | Ferroptosis effectors in mesothelioma. (A) Model for ferroptosis pathway. Promoters of ferroptosis (red) include ACSL4, NOX, and ROS, while SLC7A11, SLC7A10, MSRB2, GPX4, and AKR1C1-3 (green) are ferroptosis scavengers. ACSL4 expression is activated by YAP/TAZ while BAP1 inhibits the expression of SLC7A11. CDKN2A-encoded p16 is one of the effectors of senescence where ferroptosis is prevented by increased expression of FTH1 and IREB2 accompanied by decreased levels of ISCU. (B) "Oncoprint" analysis of ferroptosis effectors in TCGA data performed using cBioportal (www.cBioportal.org). 
1-3(AKR1C1-3) (20). These enzymes have been shown to participate in the detoxification of reactive aldehyde generated downstream of the oxidation of various PUFA.

Taking into account all this information, a mesothelial cell losing BAP1 function becomes resistant to ROS and ferroptosis, while mesothelial cells losing NF2 function become "primed" for ferroptosis, while loss of p16 expression will be associated with impaired senescence-driven ferroptosis resistance.

Loss of BAP1 is mostly associated with epithelioid histotype (21), while loss of NF2 function is mostly associated with high S-score, which identifies tumor samples with a high sarcomatoid phenotype component (22). This is consistent with the observation that cells in a mesenchymal state, which are less sensitive to chemotherapeutics, have been shown to rely on GPX4 function to avoid ferroptosis (23-25). Intriguingly, Nagai et al. observed that iron chelation did not prevent mesothelioma development in rats upon exposure to asbestos fibers, but tumor histotype shifted toward increased incidence of epithelioid compared to the sarcomatoid histotype observed in the control group (26). In the absence of accompanying genomic alteration analysis of those tumors it is not possible to know whether the two groups had a different genetic alteration profile or whether there was a plasticity response of cancer cells to the environment.

Recently, in our own model of mesothelioma development (13) we observed a significant $(p=0.008971$, FDR $=0.0145) 1.4$ fold increase of Acsl4 and a significant 74 and $91 \%$ decrease of Gpx4 ( $p=6.28 \mathrm{E}-22, \mathrm{FDR}=8.19 \mathrm{E}-21)$ and Msrb2 $(p=1.38 \mathrm{E}-$ $88, \mathrm{FDR}=4.95 \mathrm{E}-86$ ) expression, respectively, when comparing tumors to inflamed precancerous lesions. Hence, these tumors should be predisposed to ferroptosis death, as expected from their spindeloid phenotype and YAP activation. However, Slc7a11 undergoes a significant $(p=0.004227$, FDR $=0.007263) 4.7$ fold upregulation as well, consistent with the loss of one BAP1 allele. Collectively, these observations suggest that tumors with alterations in both pathways, NF2 and BAP1, which occur in a significant fraction of MPM patients according to TCGA data (3) (Figure 1B), might be more resistant to ferroptosis. However, functional studies are necessary to verify this hypothesis.

Drugs modulating ferroptosis have been recently reviewed (27). Inhibitors of GPX4, such as Ras-selective-lethal 3 (RSL3) or ML210, trigger ferroptosis, while SLC7A11 inhibiting agents, such as erastine or sorafenib, lead to glutathione depletion and endoplasmic reticulum stress. The mechanism behind sorafetinib inhibition of cysteine $\mathrm{Xc}^{-}$transporter is not clear and is possibly indirect (20). Dr. Fennell pointed to two clinical trials in mesothelioma $(28,29)$, where sorafenib was used and in

\section{REFERENCES}

1. Devan J, Janikova A, Mraz M. New concepts in follicular lymphoma biology: from BCL2 to epigenetic regulators and non-coding RNAs. Semin Oncol. (2018) 45:291-302. doi: 10.1053/j.seminoncol.2018.07.005

2. Bueno R, Stawiski EW, Goldstein LD, Durinck S, De Rienzo A, Modrusan $\mathrm{Z}$, et al. Comprehensive genomic analysis of malignant pleural mesothelioma identifies recurrent mutations, gene fusions and splicing alterations. Nat Genet. (2016) 48:407-16. doi: 10.1038/ng.3520 which objective responses were observed in only in a small proportion of unselected patients. Therefore, it will be necessary to have a translational study accompanying these trials to determine if those patients that responded had a disrupted NF2/Hippo pathway.

Relevant for the current first-line therapy of mesothelioma patients, which includes cisplatin, erastin has been shown to have a synergistic cancer cell killing effect with cisplatin in in vitro models (30).

Remarkably, in a recent study ferroptosis was observed in cells treated with some open-chain epothilones small molecules in a manner similar to that of erastin (25). Additionally, mesothelioma cell killing is iron-dependent in a novel therapeutic approach using atmospheric plasma therapy (31). Plasma is the fourth condition of physical state, in addition to solid/liquid/gas [reviewed in (32)].

Given the propensity of mesenchymal cells to be sensitive to ferroptosis induction, it is tempting to suggest that mesothelioma patients with high S-score might benefit from this novel therapy. However, a plethora of novel therapies for mesothelioma have emerged (33-35) and it might be worth assessing whether mesothelioma cells can undergo ferroptosis in vivo. Indeed, it must be noted that Carbonic anhydrase 9 (CAIX) has recently been shown to confer resistance to ferroptosis/apoptosis in malignant mesothelioma under hypoxia (36). Given that CAIX is ubiquitously highly expressed in mesothelioma $(37,38)$, this may have to be taken into account moving forwards. Because of the known effect of cisplatin on ROS generation [reviewed in (39)], it may also be of use to analyze the expression of PTSG2, encoding for COX-2, a marker of ferroptosis (40), in samples from these cisplatin-treated patients.

\section{AUTHOR CONTRIBUTIONS}

EF-B and SG wrote and approved the manuscript.

\section{FUNDING}

The work of EF-B was supported by the Swiss National Science Foundation grant 320030_182690 and the Stiftung für Angewandte Krebsforchung.

\section{ACKNOWLEDGMENTS}

We are grateful to Dr. Agata Okonska for critical reading of this manuscript. 
6. Dixon SJ, Lemberg KM, Lamprecht MR, Skouta R, Zaitsev EM, Gleason CE, et al. Ferroptosis: an iron-dependent form of nonapoptotic cell death. Cell. (2012) 149:1060-72. doi: 10.1016/j.cell.2012.03.042

7. Cheng JQ, Jhanwar SC, Klein WM, Bell DW, Lee WC, Altomare DA, et al. p16 alterations and deletion mapping of 9p21-p22 in malignant mesothelioma. Cancer Res. (1994) 54:5547-51.

8. Xio S, Li D, Vijg J, Sugarbaker DJ, Corson JM, Fletcher JA. Codeletion of p15 and p16 in primary malignant mesothelioma. Oncogene. (1995) 11:511-5.

9. Prins JB, Williamson KA, Kamp MM, Van Hezik EJ, Van der Kwast TH, Hagemeijer A, et al. The gene for the cyclin-dependent-kinase-4 inhibitor, CDKN2A, is preferentially deleted in malignant mesothelioma. Int J Cancer. (1998) 75:649-53.

10. Munoz-Espin D, Serrano M. Cellular senescence: from physiology to pathology. Nat Rev Mol Cell Biol. (2014) 15:482-96. doi: 10.1038/nrm3823

11. Masaldan S, Clatworthy SAS, Gamell C, Meggyesy PM, Rigopoulos AT, Haupt S, et al. Iron accumulation in senescent cells is coupled with impaired ferritinophagy and inhibition of ferroptosis. Redox Biol. (2018) 14:100-15. doi: 10.1016/j.redox.2017.08.015

12. Fennell D. Cancer-cell death ironed out. Nature. (2019) 572:314-5. doi: 10.1038/d41586-019-02218-y

13. Rehrauer H, Wu L, Blum W, Pecze L, Henzi T, Serre-Beinier V, et al. How asbestos drives the tissue towards tumors: YAP activation, macrophage and mesothelial precursor recruitment, RNA editing, and somatic mutations. Oncogene. (2018) 37:2645-59. doi: 10.1038/s41388-018-0153-z

14. Wallace DC, Fan W, Procaccio V. Mitochondrial energetics and therapeutics. Annu Rev Pathol. (2010) 5:297-348. doi: 10.1146/annurev.pathol.4.110807.092314

15. Hirschhorn T, Stockwell BR. The development of the concept of ferroptosis. Free Radic Biol Med. (2019) 133:130-43. doi: 10.1016/j.freeradbiomed.2018.09.043

16. Conrad M, Sato $H$. The oxidative stress-inducible cystine/glutamate antiporter, system x (c) (-): cystine supplier and beyond. Amino Acids. (2012) 42:231-46. doi: 10.1007/s00726-011-0867-5

17. Connell ND, Rheinwald JG. Regulation of the cytoskeleton in mesothelial cells: reversible loss of keratin and increase in vimentin during rapid growth in culture. Cell. (1983) 34:245-53. doi: 10.1016/0092-8674(83)90155-1

18. Shi Y, Moura U, Opitz I, Soltermann A, Rehrauer H, Thies S, et al. Role of hedgehog signaling in malignant pleural mesothelioma. Clin Cancer Res. (2012) 18:4646-56. doi: 10.1158/1078-0432.CCR-12-0599

19. Ishii T, Hishinuma I, Bannai S, Sugita Y. Mechanism of growth promotion of mouse lymphoma L1210 cells in vitro by feeder layer or 2-mercaptoethanol. $J$ Cell Physiol. (1981) 107:283-93. doi: 10.1002/jcp.1041070215

20. Dixon SJ, Patel DN, Welsch $M$, Skouta R, Lee ED, Hayano M, et al. Pharmacological inhibition of cystine-glutamate exchange induces endoplasmic reticulum stress and ferroptosis. Elife. (2014) 3:e02523. doi: 10.7554/eLife.02523

21. Yoshikawa Y, Sato A, Tsujimura T, Emi M, Morinaga T, Fukuoka $\mathrm{K}$, et al. Frequent inactivation of the BAP1 gene in epithelioidtype malignant mesothelioma. Cancer Sci. (2012) 103:868-74. doi: 10.1111/j.1349-7006.2012.02223.x

22. Blum Y, Meiller C, Quetel L, Elarouci N, Ayadi M, Tashtanbaeva D, et al. Dissecting heterogeneity in malignant pleural mesothelioma through histomolecular gradients for clinical applications. Nat Commun. (2019) 10:1333. doi: 10.1038/s41467-019-09307-6

23. Hangauer MJ, Viswanathan VS, Ryan MJ, Bole D, Eaton JK, Matov A, et al. Drug-tolerant persister cancer cells are vulnerable to GPX4 inhibition. Nature. (2017) 551:247-50. doi: 10.1038/nature24297

24. Viswanathan VS, Ryan MJ, Dhruv HD, Gill S, Eichhoff OM, Seashore-Ludlow B, et al. Dependency of a therapy-resistant state of cancer cells on a lipid peroxidase pathway. Nature. (2017) 547:453-7. doi: 10.1038/nature23007

25. Taylor WR, Fedorka SR, Gad I, Shah R, Alqahtani HD, Koranne R, et al. Smallmolecule ferroptotic agents with potential to selectively target cancer stem cells. Sci Rep. (2019) 9:5926. doi: 10.1038/s41598-019-42251-5

26. Nagai H, Okazaki Y, Chew SH, Misawa N, Yasui H, Toyokuni S. Deferasirox induces mesenchymal-epithelial transition in crocidolite-induced mesothelial carcinogenesis in rats. Cancer Prev Res. (2013) 6:1222-30. doi: 10.1158/1940-6207.CAPR-13-0244

27. Conrad M, Angeli JP, Vandenabeele P, Stockwell BR. Regulated necrosis: disease relevance and therapeutic opportunities. Nat Rev Drug Discov. (2016) 15:348-66. doi: 10.1038/nrd.2015.6

28. Dubey S, Janne PA, Krug L, Pang H, Wang X, Heinze R, et al. A phase II study of sorafenib in malignant mesothelioma: results of Cancer and Leukemia Group B 30307. J Thorac Oncol. (2010) 5:1655-61. doi: 10.1097/JTO.0b013e3181ec18db

29. Papa S, Popat S, Shah R, Prevost AT, Lal R, McLennan B, et al. Phase 2 study of sorafenib in malignant mesothelioma previously treated with platinum-containing chemotherapy. J Thorac Oncol. (2013) 8:783-7. doi: 10.1097/JTO.0b013e31828c2b26

30. Sato M, Kusumi R, Hamashima S, Kobayashi S, Sasaki S, Komiyama Y, et al. The ferroptosis inducer erastin irreversibly inhibits system xc- and synergizes with cisplatin to increase cisplatin's cytotoxicity in cancer cells. Sci Rep. (2018) 8:968. doi: 10.1038/s41598-018-19213-4

31. Shi L, Ito F, Wang Y, Okazaki Y, Tanaka H, Mizuno M, et al. Non-thermal plasma induces a stress response in mesothelioma cells resulting in increased endocytosis, lysosome biogenesis and autophagy. Free Radic Biol Med. (2017) 108:904-17. doi: 10.1016/j.freeradbiomed.2017.04.368

32. Toyokuni S. Iron addiction with ferroptosis-resistance in asbestos-induced mesothelial carcinogenesis: toward the era of mesothelioma prevention. Free Radic Biol Med. (2019) 133:206-15. doi: 10.1016/j.freeradbiomed.2018. 10.401

33. Yap TA, Aerts JG, Popat S, Fennell DA. Novel insights into mesothelioma biology and implications for therapy. Nat Rev Cancer. (2017) 17:475-88. doi: $10.1038 / \mathrm{nrc} .2017 .42$

34. de Gooijer CJ, Baas P, Burgers JA. Current chemotherapy strategies in malignant pleural mesothelioma. Transl Lung Cancer Res. (2018) 7:574-83. doi: $10.21037 /$ tlcr.2018.04.10

35. Carbone M, Adusumilli PS, Alexander HR Jr, Baas P, Bardelli F, Bononi A, et al. Mesothelioma: scientific clues for prevention, diagnosis, and therapy. CA Cancer J Clin. (2019) 69:402-29. doi: 10.3322/caac.21572

36. Li Z, Jiang L, Chew SH, Hirayama T, Sekido Y, Toyokuni S. Carbonic anhydrase 9 confers resistance to ferroptosis/apoptosis in malignant mesothelioma under hypoxia. Redox Biol. (2019) 26:101297. doi: 10.1016/j.redox.2019.101297

37. Ramsey ML, Yuh BJ, Johnson MT, Yeldandi AV, Zynger DL. Carbonic anhydrase IX is expressed in mesothelioma and metastatic clear cell renal cell carcinoma of the lung. Virchows Arch. (2012) 460:89-93. doi: 10.1007/s00428-011-1178-7

38. Capkova L, Koubkova L, Kodet R. Expression of carbonic anhydrase IX (CAIX) in malignant mesothelioma. An immunohistochemical and immunocytochemical study. Neoplasma. (2014) 61:161-9. doi: 10.4149/neo_2014_031

39. Galluzzi L, Vitale I, Michels J, Brenner C, Szabadkai G, Harel-Bellan A, et al. Systems biology of cisplatin resistance: past, present and future. Cell Death Dis. (2014) 5:e1257. doi: 10.1038/cddis.2013.428

40. Yang WS, SriRamaratnam R, Welsch ME, Shimada K, Skouta R, Viswanathan VS, et al. Regulation of ferroptotic cancer cell death by GPX4. Cell. (2014) 156:317-31. doi: 10.1016/j.cell.2013.12.010

Conflict of Interest: The authors declare that the research was conducted in the absence of any commercial or financial relationships that could be construed as a potential conflict of interest.

The handling editor declared a past co-authorship with the authors.

Copyright (C) 2019 Felley-Bosco and Gray. This is an open-access article distributed under the terms of the Creative Commons Attribution License (CC BY). The use, distribution or reproduction in other forums is permitted, provided the original author(s) and the copyright owner(s) are credited and that the original publication in this journal is cited, in accordance with accepted academic practice. No use, distribution or reproduction is permitted which does not comply with these terms. 